Avinash Kali, MS

Richard L. Q. Tang, MD

Andreas Kumar, MD

James K. Min, MD

Rohan Dharmakumar, PhD

\title{
Detection of Acute Reperfusion Myocardial Hemorrhage with Cardiac MR Imaging: T2 versus
}

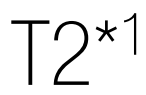

Purpose:

Materials and Methods:

Results:

Conclusion:
To evaluate $\mathrm{T} 2$ and $\mathrm{T} 2 *$ changes in acute reperfused hemorrhagic and nonhemorrhagic myocardial infarctions and to determine which technique is more suitable in the detection of intramyocardial hemorrhage at $1.5 \mathrm{~T}$.

Patient studies were approved by the institutional review board and were HIPAA compliant. Patients $(n=14$, three women) with first ST-elevation myocardial infarction underwent cardiac magnetic resonance (MR) imaging 3 days after angioplasty. T2* maps, T2 short inversion time inversion-recovery (STIR) images, and late gadolinium enhancement (LGE) images were acquired. Animal studies were approved by the institutional animal care and use committee. Canines $(n=20)$ were subjected to ischemia-reperfusion injury, and cardiac MR imaging was performed 5 days after reperfusion. T2* and T2 maps and T2 STIR and LGE images were acquired. Repeated-measures analysis of variance or the Friedman test was used to compare T2 and $\mathrm{T}^{*}$ changes in patients with hemorrhagic infarctions and those with nonhemorrhagic infarctions.

Relative to remote myocardium, mean $\mathrm{T}^{*}$ of hemorrhagic infarctions was $54 \% \pm 13$ (standard deviation) lower in patients $(15.9 \mathrm{msec} \pm 4.5$ vs $35.2 \mathrm{msec} \pm 2.1, P<.001)$ and $40 \% \pm 10$ lower in canines $(23.0 \mathrm{msec} \pm 4.0$ vs 39.3 msec $\pm 2.5, P<.001)$. Mean T2* of nonhemorrhagic infarctions was marginally elevated by $6 \% \pm 2.5(37.8 \mathrm{msec}$ $\pm 2.5, P=.021)$ in patients and by $8 \% \pm 5(44.6 \mathrm{msec}$ $\pm 4.8, P=.012)$ in canines. In contrast, mean T2 STIR signal intensity (SI) of both hemorrhagic infarctions and nonhemorrhagic infarctions was higher than that in remote myocardium both in patients (hemorrhagic: $37 \% \pm 19, P<$ .001 ; nonhemorrhagic: $78 \% \pm 27, P<.001)$ and in canines (hemorrhagic: $42 \% \pm 22, P<.001$; nonhemorrhagic: $65 \%$ $\pm 22, P<.001)$. Consistent with STIR SI findings, mean $\mathrm{T} 2$ of both hemorrhagic ( $62.0 \mathrm{msec} \pm 4.9)$ and nonhemorrhagic $(71.7 \mathrm{msec} \pm 7.3)$ infarctions in canines was elevated relative to mean T2 of remote myocardium (52.1 msec \pm 4.8$)$ by $18 \% \pm 9$ and $38 \% \pm 13$, respectively $(P<$ .001 for both).

T2* cardiac MR imaging is more suitable than T2 cardiac MR imaging in the detection and characterization of acute reperfusion myocardial hemorrhage.

${ }^{\circ}$ RSNA, 2013

Supplemental material: http://radiology.rsna.org/lookup /suppl/doi:10.1148/radiol.13122397/-/DC1 
$\mathbf{T}$ imely restoration of blood flow to ischemic myocardium can limit the extent of myocardial necrosis (1). Although the benefits of reperfusion therapy are well established, ischemia-reperfusion (I-R) injury has been shown to be associated with postinfarction ventricular arrhythmias (2), myocardial stunning (3), microvascular obstruction (MVO) (4), and lethal reperfusion injury (5). In particular, MVO resulting from I-R injury has been associated with adverse outcomes and reduced event-free survival $(6,7)$. Closely related to MVO is intramyocardial hemorrhage, which is a condition where reperfused blood extravasates into the interstitial space. Intramyocardial hemorrhage has been widely reported to be associated with larger infarctions and MVO after reperfusion both in animal models (8-11) and in humans (12-14). It is important to note that intramyocardial hemorrhage has been shown to be a significant predictor of poor left ventricular (LV) remodeling (15-18), late arrhythmogenic risk (19), and major adverse cardiovascular events (20).

Cardiac magnetic resonance (MR) imaging based on either $\mathrm{T}^{*}$-weighted $(16,18,21-24)$ or T2-weighted $(15,17$,

\section{Advances in Knowledge}

- Validated T2 and T2* values of hemorrhagic and nonhemorrhagic infarctions at $1.5 \mathrm{~T}$ are reported.

- Between T2- and T2*-based methods, only $\mathrm{T} 2 *$ maps showed the capacity to generate negative image contrast between hemorrhagic and remote myocardial territories.

- The image contrast in the detection of acute reperfusion hemorrhage at $1.5 \mathrm{~T}$ is significantly greater with $\mathrm{T} 2 *$-based cardiac MR imaging than with T2-based cardiac MR imaging.

- In nonhemorrhagic infarctions, $\mathrm{T} 2 *$ changes between infarct and remote myocardial territories were marginal, indicating that $\mathrm{T} 2 *$ is relatively insensitive to myocardial edema.
19,25-28) sequences has been used to noninvasively discriminate between hemorrhagic and nonhemorrhagic infarctions. In principle, either the T2 or the T2* approach may be used to identify the presence of hemorrhage, since both methods are sensitive to the magnetic field inhomogeneities associated with the byproducts of hemoglobin degradation (29). However, there is currently a lack of general agreement on which approach (T2 or $\mathrm{T}^{*}$ ) is most suitable for imaging acute intramyocardial hemorrhage. Since T2-based imaging is known to be highly sensitive to edema, which commonly accompanies acute reperfusion hemorrhage, the appearance of hemorrhage may be masked or reduced on T2-based images (30).

We hypothesized that $\mathrm{T} 2$ *-based imaging could provide greater acuity in the detection of reperfusion hemorrhage than could T2-weighted imaging. We tested this hypothesis in a cohort of individuals who underwent coronary revascularization after first ST-elevation myocardial infarction (STEMI) and validated our findings by using an established large-animal model of acute I-R injury. We evaluated $\mathrm{T} 2$ and $\mathrm{T}_{2}^{*}$ changes in acute reperfused hemorrhagic and nonhemorrhagic myocardial infarctions to determine which of the two techniques is more suitable for detecting intramyocardial hemorrhage at $1.5 \mathrm{~T}$.

\section{Materials and Methods}

\section{Patient Studies}

Patient studies were approved by the institutional review board and were compliant with the Health Insurance Portability and Accountability Act. After informed consent was obtained, 14 patients (three

\section{Implication for Patient Care}

- The occurrence of myocardial reperfusion hemorrhage, which has been associated with acute myocardial damage and adverse chronic remodeling, is expected to be more reliably assessed with T2* cardiac MR imaging than with T2-based cardiac MR imaging. women; mean age, 56 years \pm 9 [standard deviation]) with first STEMI and successful percutaneous coronary intervention (PCI) (Thrombolysis in Myocardial Infarction flow of 3 after PCI) were prospectively enrolled and studied within 3 days after PCI. Patients with contraindications to MR imaging were excluded from study recruitment. Electrocardiographically triggered breath-hold $\mathrm{T}^{*}$-weighted multiple gradient-recalled echo, T2weighted short inversion time inversionrecovery (STIR), and T1-weighted late gadolinium enhancement (LGE) images of contiguous short-axis sections covering the entire left ventricle (LV) were acquired with a 1.5-T clinical MR imager (Magnetom; Avanto, Siemens Healthcare, Erlangen, Germany). Typical imaging parameters used to acquire different MR images are provided in Appendix E1 [online].

\section{Proof-Of-Concept Studies in Canines}

Twenty-five canines (weight range, $20-25 \mathrm{~kg}$ ) were studied according to the protocols approved by the

\section{Published online before print}

10.1148/radiol.13122397 Content code: CA

Radiology 2013; 269:387-395

\section{Abbreviations:}

$\mathrm{I}-\mathrm{R}=$ ischemia-reperfusion

$\mathrm{LGE}=$ late gadolinium enhancement

$\mathrm{LV}=$ left ventricle

MV0 = microvascular obstruction

$\mathrm{PCl}=$ percutaneous coronary intervention

$\mathrm{ROI}=$ region of interest

$\mathrm{SI}=$ signal intensity

STEMI = ST-elevation myocardial infarction

$\mathrm{STIR}=$ short inversion time inversion recovery

\section{Author contributions:}

Guarantors of integrity of entire study, A. Kali, R.D.; study concepts/study design or data acquisition or data analysis/interpretation, all authors; manuscript drafting or manuscript revision for important intellectual content, all authors; approval of final version of submitted manuscript, all authors; literature research, all authors; clinical studies, R.L.Q.T., A. Kumar, J.K.M., R.D.; experimental studies, A. Kali, R.L.Q.T., A. Kumar, R.D.; statistical analysis, A. Kali, R.L.Q.T., J.K.M.; and manuscript editing, all authors

\section{Funding:}

This research was supported by the National Institutes of Health (grant R01 HL091989).

Conflicts of interest are listed at the end of this article. 
institutional animal care and use committee at Northwestern University. Detailed descriptions of animal preparation and the I-R protocol, as well as the study timeline (Fig E1 [online]), are provided in Appendix E1 (online). Baseline cardiac MR imaging was performed on day 0 . On day 2 , a hydraulic occluder was implanted around the left anterior descending coronary artery. On day 9, each animal was randomly assigned to one of two experimental groups: infarct $(n=20)$ or control $(n=5)$. The infarct group underwent 3 hours of no-flow ischemia followed by reperfusion. Cardiac MR imaging was performed in the infarct group again on day 14 after baseline imaging (5 days after I-R injury). The control group did not undergo the I-R protocol, and cardiac MR imaging of these animals was performed on day 14 after baseline imaging to match the imaging time points of the infarct group.

Cardiac MR studies were performed with a 1.5-T clinical MR imaging system (Magnetom Espree; Siemens Healthcare). Electrocardiographically triggered, breath-hold contiguous short-axis images covering the entire LV were acquired by using $\mathrm{T} 2$ * weighted multiple gradient-recalled echo, T2-weighted STIR, T2-prepared steady-state free precession, and LGE imaging. Typical imaging parameters used to acquire different images are provided in Appendix E1 (online).

Animals were humanely euthanized after the 14-day MR study. The hearts were excised and cut into 8-10-mm-thick short-axis slices. Infarcted areas were delineated by using triphenyltetrazolium chloride staining. Microscopic histopathologic analyses of the representative samples of infarcted and remote myocardium were assessed with hematoxylin-eosin and Perl staining. Infarctions were considered hemorrhagic if Perl staining showed the presence of iron and if hematoxylin-eosin staining showed the presence of extravasated red blood cells within the infarcted zones. Otherwise, infarctions were considered nonhemorrhagic.

\section{Image Analysis}

Motion-corrected inline T2 maps were obtained by using T2-prepared steadystate free precession images. T2* maps were generated by fitting the multiple gradient-recalled echo images to a monoexponential decay model. All image analyses were performed with validated image processing software $\left(\mathrm{cmr}^{42}\right.$; Circle Cardiovascular Imaging, Calgary, Alberta, Canada). The images were evaluated in consensus by two expert reviewers (A.Kali, R.D.), each with more than 5 years of experience evaluating cardiac MR images. The reviewers were blinded to any other patient or canine data. Remote myocardium was identified as the region that showed no hyperintensity on LGE images. Infarcted myocardium was defined as the region with mean signal intensity (SI) that is at least 5 standard deviations greater than that of a reference region of interest (ROI) drawn in remote myocardium (31) (Fig E2 [online]).

In patients, infarctions were considered hemorrhagic if they contained hypointense cores on the $\mathrm{T} 2{ }^{*}$-weighted image acquired at an echo time of 13.7 msec and a repetition time of $15.8 \mathrm{msec}$ with a mean SI that is at least 2 standard deviations below that of the reference ROI. Infarctions were considered nonhemorrhagic if they were negative for hemorrhage on $\mathrm{T}^{*}$-weighted images. ROIs were drawn around the hemorrhagic myocardium on $\mathrm{T} 2{ }^{*}$-weighted images and copied onto the corresponding T2 STIR images. Mean T2 STIR SI and mean $\mathrm{T}^{*}$ were measured for remote myocardium, as well as for hemorrhagic and nonhemorrhagic infarctions.

Canines from the infarct group were classified as having either hemorrhagic or nonhemorrhagic infarctions based on ex vivo histopathologic analysis, as explained previously. In vivo cardiac MR images were then retrospectively analyzed based on this classification. Hemorrhagic myocardium was identified as hypointense cores within infarcted territories on the T2*-weighted image acquired with a 15.4-msec echo time, a 21.0-msec repetition time, and a mean SI that is at least 2 standard deviations less than that of the reference ROI. ROIs were drawn around the hemorrhagic myocardium on
$\mathrm{T} 2{ }^{*}$-weighted images and copied onto the corresponding T2 maps and T2 STIR images. For canines from the control group, mean T2 and T2* values of the entire LV were measured from day 0 (baseline) and day 14 MR studies. For the canines from the infarct group, mean baseline $\mathrm{T} 2$ and $\mathrm{T}^{*}$ values of the entire $\mathrm{LV}$ were measured from the day 0 MR study. For the same animals from the infarct group, mean T2, T2* and STIR SI values for the ROIs within remote, nonhemorrhagic, and hemorrhagic myocardium were measured from the day 14 MR study (5 days after I-R injury).

\section{Statistical Analyses}

Statistical analyses were performed by using Stata 10.1 software (StataCorp, College Station, Tex). Categorical variables were expressed as numbers and percentages of animals or patients and compared by using the $\chi^{2}$ test. Normality of continuous data was determined by using the Shapiro-Wilk test and quantilequantile plots. Continuous variables with normal distribution were compared by using mixed-model analysis of variance with Tukey post hoc analysis. ROIs within each heart were entered as fixed effects, while the animals or patients were entered as random effects. Repeated measures from each heart were nested for analysis. Continuous variables with nonnormal distribution were compared by using the nonparametric Friedman test. Pairwise comparisons for nonnormal data were also performed by using the Mann-Whitney $U$ test. Bonferroni corrections were used to adjust the significance level for multiple comparisons.

\section{Results}

\section{Patient Studies}

Clinical features of patients with STEMI enrolled in this study are shown in Table $1 . \mathrm{T}^{*}$-weighted images, in conjunction with LGE images, showed hemorrhagic infarctions in seven patients (three lateral, three anteroseptal, and one inferior lateral infarction) and nonhemorrhagic infarctions in seven patients (three lateral, two anteroseptal, and two inferior lateral infarctions). Representative T2* maps and T2 STIR 
and LGE images acquired on day 3 after PCI in one patient with hemorrhagic infarction and one patient with nonhemorrhagic infarction are shown in Figure 1. Visually evident $\mathrm{T}_{2}$ * decreases and STIR SI increases were observed within hemorrhagic infarctions on T2* maps and T2 STIR images, respectively. Hypointense cores within hyperintense edematous territories that were positive for LGE were seen on T2 STIR images of hemorrhagic infarctions. Hemorrhage was always accompanied by persistent MVO on LGE images. Marked T2* and STIR SI increases were seen within nonhemorrhagic infarctions.

Mean T2* values of the remote, hemorrhagic, and nonhemorrhagic myocardium measured in patients on day 3 after PCI are shown in Table 2. When compared with the remote myocardium, mean $\mathrm{T}^{*}$ of hemorrhagic infarction was $54 \% \pm 13$ lower $(P<.001)$, while mean STIR SI of hemorrhagic myocardium was $37 \% \pm 19$ higher $(P<.001)$. Mean $\mathrm{T}^{*}$ of nonhemorrhagic myocardium showed a marginal increase of $6 \% \pm 2.5(P=.021)$, while mean STIR SI was significantly increased by $78 \% \pm 27(P<.001)$. Mean percentage change in STIR SI of hemorrhagic infarction with respect to remote myocardium was significantly lower than that of nonhemorrhagic infarction $(P<$ $.001)$. In all, $38(88 \%)$ of the 43 imaging sections that were positive for hemorrhage on $\mathrm{T} 2{ }^{*}$-weighted images showed hypointense cores within hyperintense edematous territories on the corresponding T2-weighted STIR images.

\section{Animal Studies}

After discounting for premature death during reperfusion $(n=2)$ and occluder dysfunction $(n=4), 14$ animals from the infarct group were available for analysis. Nine animals from the infarct group sustained hemorrhagic infarctions, while five animals sustained nonhemorrhagic infarctions, as verified with ex vivo histopathologic analysis. Control animals did not sustain any infarction throughout the study.

Representative $\mathrm{T} 2 *$ maps, T2 maps, T2 STIR images, and LGE images acquired on day 14 with MR imaging from a canine in the control group and from two

\section{Table 1}

\section{Clinical Features of Patients with STEMI}

\begin{tabular}{lccc} 
Parameter & Hemorrhagic Group $(n=7)$ & Nonhemorrhagic Group $(n=7)$ & PValue \\
\hline Age $(y)^{\star}$ & $56(47-59)$ & $57(56-66)$ & .28 \\
Male sex & $6(86)$ & $3(43)$ & .09 \\
Diabetes mellitus & $3(43)$ & $2(29)$ & .58 \\
Smoking history & $5(71)$ & $1(14)$ & $.03^{\dagger}$ \\
Hypertension & $2(29)$ & $2(29)$ & $>.99$ \\
Hyperlipidemia & $2(29)$ & $1(14)$ & .51 \\
Time from symptoms to reperfusion $(\mathrm{h})^{\star}$ & $4.8(3.4-7.3)$ & $3.7(3.2-4.0)$ & .25 \\
TIMl flow before PCI & & & $\ldots$ \\
$\quad$ Grade 0 & $3(43)$ & $3(43)$ & .76 \\
Grade 1 & $2(29)$ & $1(14)$ & $\ldots$ \\
Grade 2 & $2(29)$ & $3(43)$ & $\mathrm{NA}$ \\
\hline Grade 3 & $7(100)$ & $7(100)$ & \\
\hline
\end{tabular}

Note.-Unless otherwise indicated, data are numbers of patients, and data in parentheses are percentages. NA = not applicable, $\mathrm{PCl}=$ percutaneous coronary intervention, $\mathrm{TIMI}=$ thrombolysis in myocardial infarction .

* Data are medians, and data in parentheses are the interquartile range.

${ }^{\dagger}$ Significant difference.

canines in the infarct group (one with hemorrhagic infarction and one with nonhemorrhagic infarction) are shown in Figure 2. Consistent with the patient studies, all canines with hemorrhagic infarctions showed distinct hypointense cores on at least one of the in vivo $\mathrm{T} 2$ * maps, T2 maps, and T2 STIR images acquired on day 14. The hypointense cores were always surrounded by hyperintense edematous territories on both $\mathrm{T} 2$ maps and T2 STIR images. Hemorrhage was always accompanied by persistent MVO on LGE images. On the other hand, canines with nonhemorrhagic infarctions did not have any hypointense cores within infarcted territories on any of the in vivo T2* maps, T2 maps, or T2 STIR images acquired on day 14. A marginal increase in $\mathrm{T}^{*}$ was visually evident in nonhemorrhagic infarctions, while T2 and STIR SI were substantially increased.

Mean T2 and T2* values in the control and infarct groups measured on days 0 and 14 are shown in Table 3 . Among the control patients, mean baseline $\mathrm{T}^{*}$ and $\mathrm{T} 2$ values measured with MR imaging at day 0 were not significantly different from their respective mean values measured at day $14(P=.19)$. Similarly, within the infarct group, mean baseline $\mathrm{T}^{*}$ and $\mathrm{T} 2$ values of the entire
LV measured with MR imaging at day 0 were not significantly different from the respective values of remote myocardium measured at day $14(P=.18)$. In contrast, mean $\mathrm{T}_{2}^{*}$ of hemorrhagic territories at MR imaging on day 14 was significantly lower than the mean baseline $\mathrm{T} 2^{*}$ of the entire $L V$ measured prior to I-R injury $(P<.001)$, while the mean T2 of hemorrhagic territories at MR imaging on day 14 was significantly higher than the mean baseline T2 of the entire $\mathrm{LV}$ prior to I-R injury $(P<.001)$. Mean T2* and T2 values of nonhemorrhagic territories obtained at MR imaging on day 14 were significantly higher than their respective baseline measures prior to I-R injury ( $P$ $<.001$ for both). In all, $42(94 \%)$ of the 45 imaging sections that were positive for hemorrhage on $\mathrm{T} 2{ }^{*}$-weighted images showed hypointense cores within hyperintense edematous territories on the corresponding T2 maps, while 41 (91\%) of the 45 sections that were positive for hemorrhage showed hypointense cores on T2 STIR images.

Across all terminal (day 14) MR studies performed within the infarct group, mean $\mathrm{T}^{*}$ of hemorrhagic infarctions was $40 \% \pm 10$ lower than mean $\mathrm{T}^{*}$ of remote myocardium $(P<.001)$. However, mean T2 and STIR SI of hemorrhagic 


\section{Figure 1}
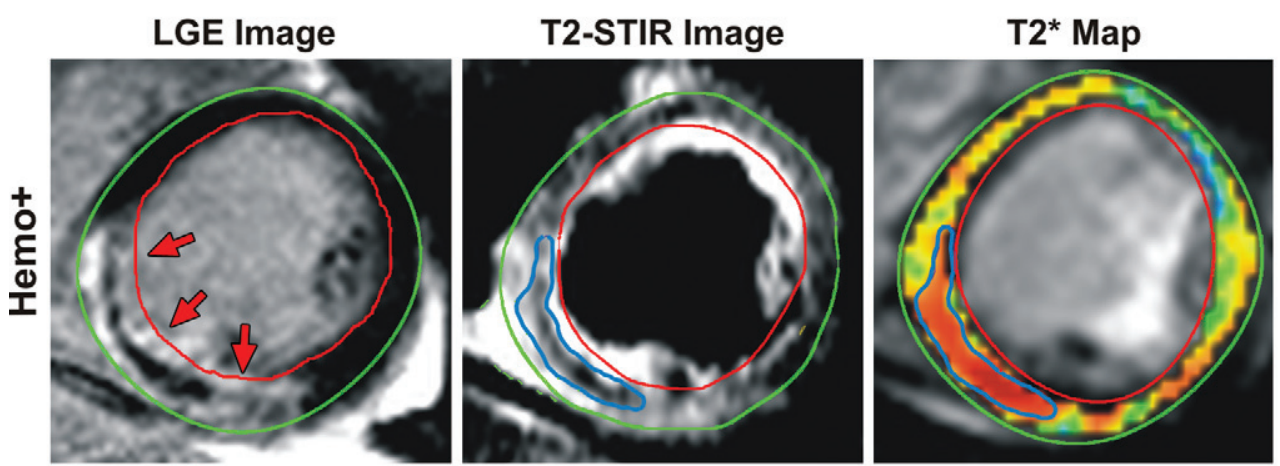

$80 \mathrm{~ms}$
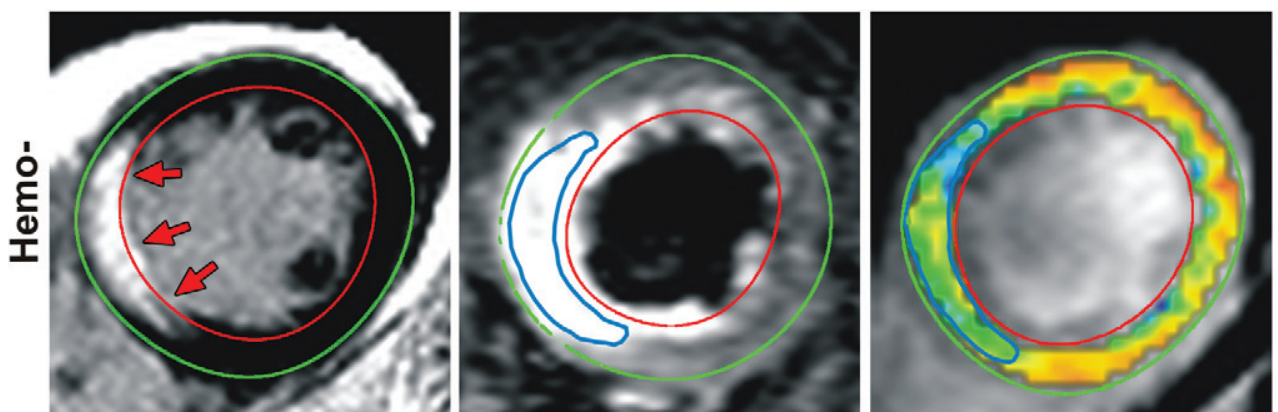

Figure 1: Cardiac MR-based detection of acute hemorrhagic (Hemo+) and nonhemorrhagic (Hemo-) myocardial infarctions in patients. Representative LGE images (inversion recovery-prepared fast low-angle shot sequence; $0.1 \mathrm{mmol}$ of gadopentetate dimeglumine per kilogram of body weight; repetition time/echo time msec/inversion time msec, two R-R intervals/3.32/250; flip angle, $25^{\circ}$ ), T2 STIR images (two or three R-R intervals/61/170), and T2* maps (multigradient echo; repetition time msec/echo time msec, 15.8/2.6, $4.8,7.0,9.3,11.5$, and 13.7 ; flip angle, $10^{\circ}$ ) acquired on day 3 after angioplasty in a 49-year-old man with hemorrhagic infarction and a 57-year-old man with nonhemorrhagic infarction. LGE images enabled us to confirm the presence of infarction (arrows) in both patients. Marked T2* decreases were observed within hemorrhagic territories, while hypointense cores were observed within the hyperintense edematous territories on T2 STIR images. Marked hyperintensities were observed within the nonhemorrhagic territories on both T2* maps and T2 STIR images.

\section{Table 2}

\begin{tabular}{|c|c|c|c|}
\hline Parameter & Remote Myocardium & $\begin{array}{l}\text { Hemorrhagic Group } \\
(n=7)\end{array}$ & $\begin{array}{l}\text { Nonhemorrhagic Group } \\
(n=7)\end{array}$ \\
\hline $\mathrm{T}^{\star}{ }^{\star}$ (msec) & $35.2 \pm 2.1$ & $15.9 \pm 4.5$ & $37.8 \pm 2.5$ \\
\hline $\begin{array}{l}\text { Change in } \mathrm{T}^{*} \text { with respect to } \\
\text { remote myocardium (\%) }\end{array}$ & $\ldots$ & $-54.4 \pm 13.3$ & $6 \pm 2.5$ \\
\hline $\begin{array}{l}\text { Change in T2 STIR signal } \\
\text { intensity with respect to } \\
\text { remote myocardium (\%) }\end{array}$ & $\ldots$ & $37.2 \pm 19.4$ & $78.2 \pm 37.4$ \\
\hline
\end{tabular}

Note.-Data are mean \pm standard deviation.

territories were increased by $17 \% \pm 9$ $(P<.001)$ and $42 \% \pm 22(P<.001)$, respectively, when compared with remote myocardium. For hemorrhagic infarctions, mean percentage change in STIR SI was significantly higher than that in T2 $(P<.001)$. When compared with remote myocardium, mean $\mathrm{T}^{*}$ of nonhemorrhagic territories was increased by a marginal $8 \% \pm 5(P=$ .012), while T2 and STIR SI of nonhemorrhagic infarctions were substantially increased ( $P<.001$ for both). Similar to hemorrhagic territories, mean percentage change of STIR SI of nonhemorrhagic territories was greater than mean percentage change in T2 $(P<$ .001). When compared with nonhemorrhagic infarctions, mean percentage changes in STIR SI and T2 were significantly lower in the presence of hemorrhage $(P<.001)$.

Representative histopathologic images in a control animal and in animals with hemorrhagic and nonhemorrhagic infarctions are shown in Figure 3. Triphenyltetrazolium chloride staining enabled us to confirm the presence 
Figure 2
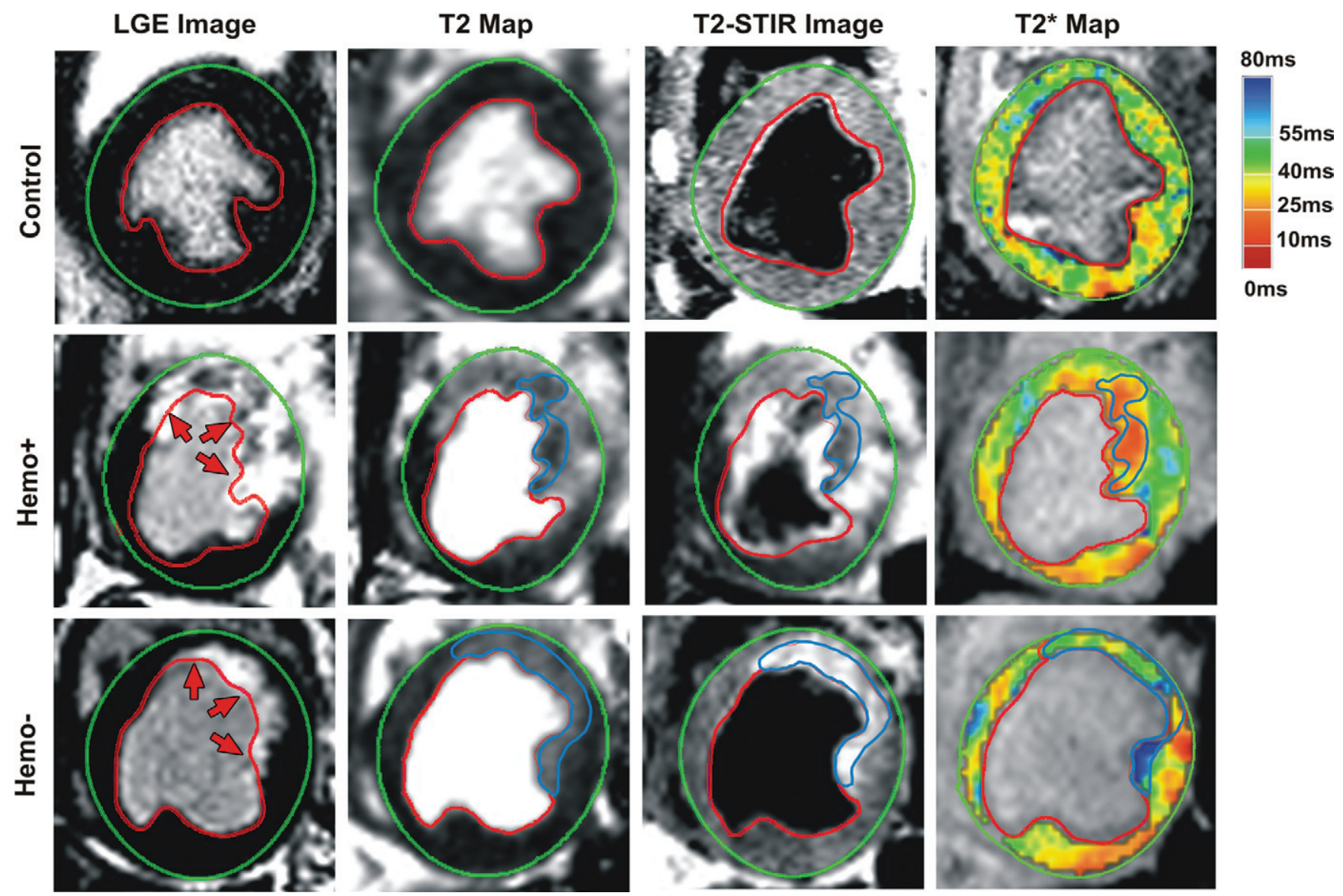

Figure 2: Cardiac MR-based detection of acute hemorrhagic (Hemo+) and nonhemorrhagic (Hemo-) myocardial infarctions in canines. Representative LGE images (inversion recovery-prepared steady-state free precession sequence; $0.1 \mathrm{mmol} / \mathrm{kg}$ gadopentetate dimeglumine; 3.75/1.75/230; flip angle, 40), T2 maps (T2prepared steady-state free precession sequence; 2.2/1.1; T2 preparation time, 0, 24, and $55 \mathrm{msec}$; flip angle, $70^{\circ}$ ), T2 STIR images (2-3 R-R intervals/64/170), and $\mathrm{T}^{\star}$ maps (multigradient echo sequence; $21 / 3.4,6.4,9.4,12.4,15.4$, and 18.4 ; flip angle, $12^{\circ}$ ) acquired 5 days after I-R injury are shown in a control animal and animals with hemorrhagic and nonhemorrhagic infarctions. Control animals did not sustain any myocardial infarction, as evidenced by LGE images. Corresponding T2 map, T2 STIR image, and T2* map did not show any distinct signal features generally observed in the presence of an acute myocardial infarction. LGE images enabled us to confirm the presence of myocardial infarctions (arrows) in animals subjected to I-R injury. Marked T2* decreases were observed in hemorrhagic territories, while hypointense cores were observed in hyperintense edematous territories on T2 maps and T2 STIR images. Nonhemorrhagic territories showed marked hyperintensity on T2 maps and T2 STIR images, while marginal T2* increases were observed on T2* maps.

of myocardial infarctions in animals subjected to I-R injury (Fig $3, B$ and $C$ ) but not in the control animals (Fig 3, A). Notably, triphenyltetrazolium chloride staining of hemorrhagic territories (Fig 3, B) showed gross bleeding within the infarct core, while no such gross bleeding was observed within triphenyltetrazolium chloride-stained nonhemorrhagic territories (Fig 3, $C)$. Hematoxylin-eosin-stained images of both hemorrhagic (Fig 3, B1) and nonhemorrhagic (Fig 3, C1) territories showed extensive myocardial damage accompanied by extensive inflammatory response. Also, hematoxylin-eosinstained images of hemorrhagic (but not nonhemorrhagic) territories showed presence of eosin-stained extravasated red blood cells in the interstitium. Perl staining showed substantial localized iron within hemorrhagic territories (Fig $3, B 2$ ) but negligible or no iron within nonhemorrhagic territories (Fig 3, C2). Microstructural histopathologic features of remote myocardium obtained in canines with hemorrhagic and nonhemorrhagic infarctions (Fig 3, B3, B4, $C 3$, and $C 4$ ) were similar to those of viable myocardium obtained in a control animal (Fig 3, A1 and A2).

\section{Discussion}

Recent studies have shown that intramyocardial hemorrhage may be one of the strongest predictors of adverse outcome (15-20). This has generated substantial interest in understanding the effects of reperfusion hemorrhage 


\section{Table 3}

Mean T2*, T2, and T2 STIR SI Changes Associated with Acute Reperfused Hemorrhagic and Nonhemorrhagic Myocardial Infarctions in Canines on Day 5 after I-R Injury

\begin{tabular}{|c|c|c|c|c|c|c|}
\hline \multirow[b]{2}{*}{ Parameter } & \multicolumn{2}{|c|}{ Control Group $(n=5)$} & \multicolumn{4}{|c|}{ Infarct Group ( $n=9$ ) } \\
\hline & Day 0 & Day 14 & Day 0 & Day 14 & Day 14 & Day 14 \\
\hline Tissue type & Whole LV & Whole LV & Whole LV & Remote & Hemorrhagic & Nonhemorrhagic \\
\hline T2 (msec) & $50.9 \pm 4.7$ & $51.4 \pm 4.7$ & $52.1 \pm 4.8$ & $52.2 \pm 4.7$ & $62.0 \pm 4.9$ & $71.7 \pm 7.3$ \\
\hline $\mathrm{T}^{\star}{ }^{\star}$ (msec) & $38.6 \pm 2.0$ & $39.7 \pm 1.9$ & $38.7 \pm 2.0$ & $39.3 \pm 2.5$ & $23.0 \pm 4.0$ & $44.6 \pm 4.8$ \\
\hline $\begin{array}{l}\text { Change in } \mathrm{T}^{*} \text { with respect to remote } \\
\text { myocardium (\%) }\end{array}$ & $\ldots$ & $\ldots$ & $\ldots$ & $\ldots$ & $-40.1 \pm 10.0$ & $8.0 \pm 5.0$ \\
\hline $\begin{array}{l}\text { Change in T2 with respect to remote } \\
\text { myocardium (\%) }\end{array}$ & $\ldots$ & $\ldots$ & $\ldots$ & $\ldots$ & $17.7 \pm 9.2$ & $38.4 \pm 12.9$ \\
\hline $\begin{array}{c}\text { Change in T2 STIR signal intensity with } \\
\text { respect to remote myocardium (\%) }\end{array}$ & $\ldots$ & $\ldots$ & $\ldots$ & $\ldots$ & $42.2 \pm 22.9$ & $64.5 \pm 22.4$ \\
\hline
\end{tabular}

Note.-Data are mean \pm standard deviation. In the infarct group, day 14 was 5 days after I-R injury.

on acute tissue damage and postinfarction remodeling. To facilitate and guide these investigations, it is imperative to ensure that the optimal imaging strategy is used. On the basis of patient and proof-of-concept animal studies, we showed that acute reperfusion myocardial hemorrhage is better characterized with $\mathrm{T} 2$-based cardiac MR imaging than it is with T2-based cardiac MR imaging. This observation is consistent with previous reports, which have suggested that hemorrhage and edema have counteracting influences on T2 (and T2-weighted) images $(16,30,32)$. It is expected that our findings here can be instrumental in furthering our understanding of the role of reperfusion hemorrhage in patients. In particular, the ability to define $\mathrm{T} 2 *$ values can help stratify the extent of hemorrhagic tissue damage.

Degradation byproducts of hemorrhage cause local magnetic field inhomogeneities that result in SI loss on T2- and T2*-based images (29). In T2-weighted imaging, refocusing radiofrequency pulses partially reverse the loss of phase coherence (or SI loss), leading to a reduced sensitivity to the presence of hemorrhage. In addition, T2-based approaches are highly sensitive to myocardial edema, which is associated with acute myocardial infarction. On the contrary, the absence of refocusing radiofrequency pulses in $\mathrm{T} 2 *$-based imaging leads to maximal loss of phase coherence within each echo time. Moreover, as shown in this study, T2* cardiac MR imaging is relatively insensitive to edema. These biophysical underpinnings support our findings that, both in patients and in canines, only T2* cardiac MR imaging is capable of generating negative image contrast relative to remote or healthy myocardium, while T2-based approaches have a reduced sensitivity to hemorrhage. The implications are that in cases of extensive edema, hemorrhagic volumes may be vastly underestimated or, in more extreme cases, entirely invisible and undetectable with T2 cardiac MR imaging. Moreover, partial volume effects (8-10-mm-thick imaging sections) may further limit the detection of small hemorrhages with T2-based cardiac MR imaging in comparison with $\mathrm{T} 2{ }^{*}$-based cardiac MR imaging.

Both T2- and $\mathrm{T}^{*}$-weighted imaging are limited by surface coil bias and the ensuing qualitative nature of image interpretation. In particular, dark-blood T2weighted techniques, such as T2 STIR, are affected by unsuppressed slow-flowing blood signal adjacent to the infarcted myocardium and myocardial signal loss due to through-plane motion, both of which may hamper specificity and sensitivity. T2 mapping, being quantitative in nature, can be used to overcome many of the issues that face T2-weighted imaging. However, the dependence of T2 values on interecho spacing (33) and their nonmonotonic relationship with the size of the paramagnetic agent (34) complicate absolute characterization of hemorrhage on the basis of T2. Our study showed that T2-based approaches may have adequate sensitivity in the detection of acute reperfusion myocardial hemorrhage despite these limitations. These observations are consistent with previous reports $(16,28)$.

In summary, the relative insensitivity of $\mathrm{T} 2 *$ cardiac MR imaging to edema but its strong sensitivity to byproducts of hemorrhage make this technique more suitable for the characterization of acute reperfusion myocardial hemorrhage. The counteracting effects of edema and hemorrhage and the refocusing effects of $\mathrm{T} 2$ cardiac MR imaging make hemorrhage less conspicuous on T2 maps and T2weighted images.

This study had certain limitations. First, the sample sizes in this translational study were relatively small. However, given the large $\mathrm{T} 2$ and $\mathrm{T} 2$ * changes in hemorrhagic and nonhemorrhagic infarctions, our retrospective analysis showed that the minimum statistical power among all our comparisons was 0.81 . The sample sizes in our study are comparable to those in other studies $(23,28)$. Second, T2 maps were not acquired in patients because of imaging time constraints; instead, T2 STIR images were acquired. Despite this limitation, the animal studies appear to 


\section{Figure 3}
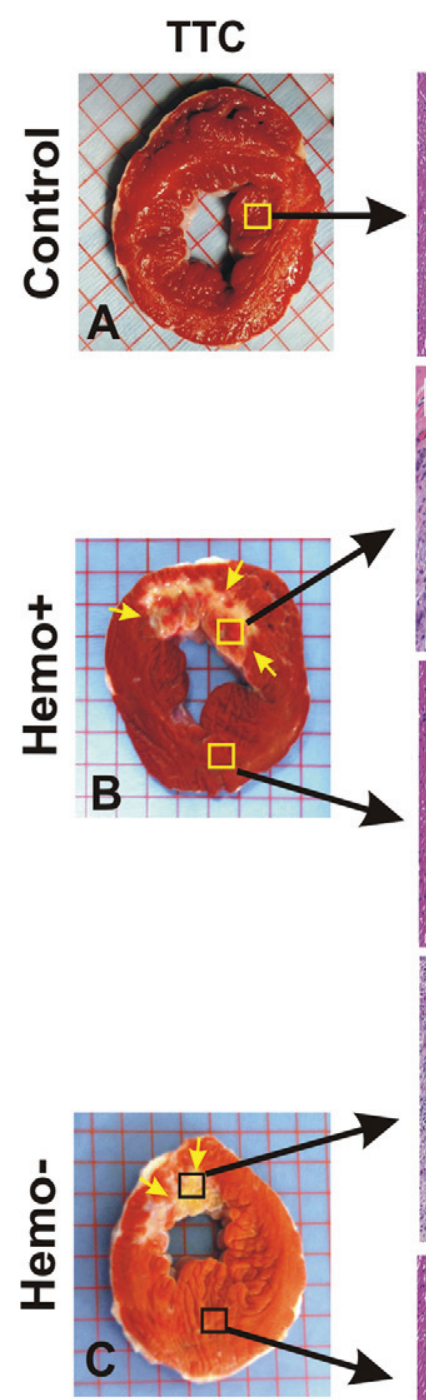

$H \& E$
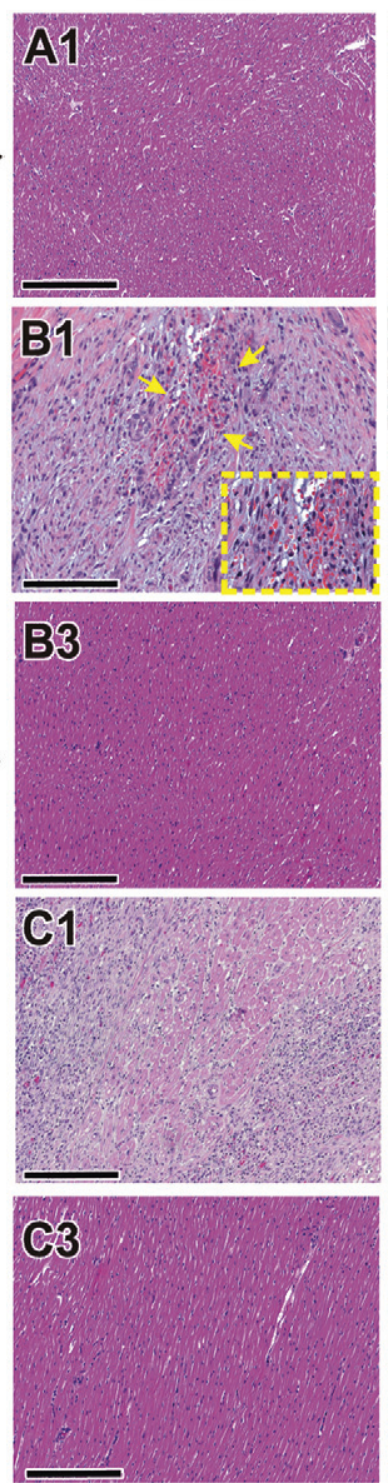

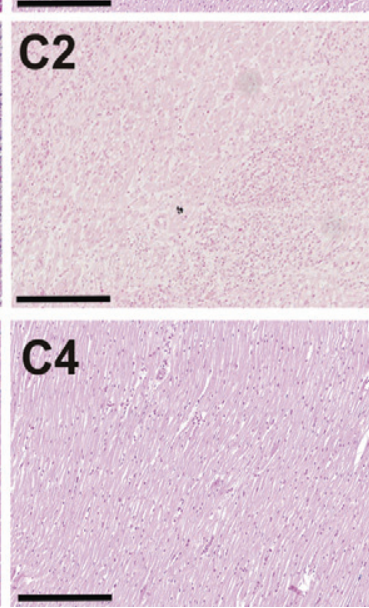

Figure 3: Histopathologic validation of acute hemorrhagic and nonhemorrhagic myocardial infarctions in canines. Representative histopathologic images obtained from an animal in the control group and two animals in the infarct group (one with hemorrhagic [Hemo+] and one with nonhemorrhagic [Hemo-] infarction) are shown. Triphenyltetrazolium chloride staining enabled us to confirm the presence of infarctions within the infarct group. Corresponding hematoxylin-eosin (H\&E) staining of hemorrhagic and nonhemorrhagic territories showed diffuse necrosis with massive infiltration of inflammatory cells (arrows and insets). Hematoxylin-eosin staining of hemorrhagic territories also showed interstitial extravasation of red blood cells into the infarcted territories. Perl staining enabled us to confirm the presence of localized iron in hemorrhagic territories (arrows and inset) but not in nonhemorrhagic territories. Scale bar $=200 \mu \mathrm{m}$.

show that there is negligible difference between the characteristic changes in $\mathrm{T} 2$ - or T2-weighted signal in remote or healthy myocardium and hemorrhagic or nonhemorrhagic territories. Third, although histologic validation of hemorrhagic infarctions identified on $\mathrm{T} 2$ * cardiac MR images was performed in canines, it could not be performed in patients, as they all survived the entire study. Fourth, the postreperfusion MR imaging time points were slightly different in patients (day 3) and canines (day 5 ). This difference stemmed from the logistical difficulties of not being able to identically match the cardiac MR studies to the postreperfusion period of infarction. Fourth, our study does not provide standard diagnostic accuracy measures for $\mathrm{T} 2$ - and $\mathrm{T} 2$ *-based cardiac MR imaging, primarily because of the subjective nature of registering the in vivo cardiac MR images with the ex vivo heart slices. Fifth, despite the sensitivity of $\mathrm{T}^{*}$ cardiac MR imaging for iron (35), it is also sensitive to offresonance artifacts arising from bulk magnetic susceptibility differences at the heart-lung interface. These artifacts can particularly affect the $\mathrm{T} 2$ *-based diagnosis of acute hemorrhagic infarctions in the inferolateral walls of the LV. Because T2* loss from off-resonance artifacts and intramyocardial hemorrhage originate from opposite sides of the myocardial wall (ie, epicardial wall for off-resonance artifacts and endocardial wall for intramyocardial hemorrhage) and because LGEs are an indication for infarction, it is possible to carefully discriminate between hemorrhage and off-resonance artifacts.

Disclosures of Conflicts of Interest: A. Kali No relevant conflicts of interest to disclose. R.L.Q.T. No relevant conflicts of interest to disclose. A. Kumar No relevant conflicts of interest to disclose. J.K.M. Financial activities related to the present article: received substan tial research support from the National Heart, Lung, and Blood Institute and GE Healthcare and modest research support from Phillips Healthcare and Vital Images. Financial activities not related to the present article: is a member of the speaker's bureau and medical advisory board of GE Healthcare. Other relationships: none to disclose. R.D. Financial activities related to the present article: received substantial research support from the National Heart, Lung, and Blood Institute and in-kind research support from Siemens Healthcare. Financial activities not related to the present article: none to disclose. Other relationships: none to disclose.

\section{References}

1. Jennings RB, Sommers HM, Smyth GA, Flack HA, Linn H. Myocardial necrosis induced by 
temporary occlusion of a coronary artery in the dog. Arch Pathol 1960;70:68-78.

2. Manning AS, Hearse DJ. Reperfusion-induced arrhythmias: mechanisms and prevention. J Mol Cell Cardiol 1984;16(6):497518.

3. Braunwald E, Kloner RA. The stunned myocardium: prolonged, postischemic ventricular dysfunction. Circulation 1982; 66(6): 1146-1149.

4. Kloner RA, Ganote CE, Jennings RB. The "no-reflow" phenomenon after temporary coronary occlusion in the dog. J Clin Invest 1974;54(6):1496-1508.

5. Kloner RA. Does reperfusion injury exist in humans? J Am Coll Cardiol 1993;21(2): $537-545$.

6. Ito H, Maruyama A, Iwakura K, et al. Clinical implications of the 'no reflow' phenomenon. a predictor of complications and left ventricular remodeling in reperfused anterior wall myocardial infarction. Circulation 1996;93(2):223-228

7. Wu KC, Zerhouni EA, Judd RM, et al. Prognostic significance of microvascular obstruction by magnetic resonance imaging in patients with acute myocardial infarction. Circulation 1998;97(8):765-772.

8. Bresnahan GF, Roberts R, Shell WE, Ross $\mathrm{J} \mathrm{Jr}$, Sobel BE. Deleterious effects due to hemorrhage after myocardial reperfusion. Am J Cardiol 1974;33(1):82-86.

9. Fishbein MC, Y-Rit J, Lando U, Kanmatsuse K, Mercier JC, Ganz W. The relationship of vascular injury and myocardial hemorrhage to necrosis after reperfusion. Circulation 1980;62(6):1274-1279.

10. Higginson LA, Beanlands DS, Nair RC, Temple V, Sheldrick K. The time course and characterization of myocardial hemorrhage after coronary reperfusion in the anesthetized dog. Circulation 1983;67(5):10241031.

11. Garcia-Dorado D, Théroux P, Solares J, et al. Determinants of hemorrhagic infarcts. histologic observations from experiments involving coronary occlusion, coronary reperfusion, and reocclusion. Am J Pathol 1990;137(2):301-311.

12. Fujiwara H, Onodera T, Tanaka M, et al. A clinicopathologic study of patients with hemorrhagic myocardial infarction treated with selective coronary thrombolysis with urokinase. Circulation 1986;73(4):749-757.

13. Asanuma T, Tanabe K, Ochiai K, et al. Relationship between progressive microvascular damage and intramyocardial hemorrhage in patients with reperfused anterior myocardial infarction: myocardial contrast echocar- diographic study. Circulation 1997;96(2): 448-453.

14. Beek AM, Nijveldt R, van Rossum AC. Intramyocardial hemorrhage and microvascular obstruction after primary percutaneous coronary intervention. Int J Cardiovasc Imaging 2010;26(1):49-55.

15. Ganame J, Messalli G, Dymarkowski S, et al. Impact of myocardial haemorrhage on left ventricular function and remodelling in patients with reperfused acute myocardial infarction. Eur Heart J 2009;30(12):14401449 .

16. O’Regan DP, Ariff B, Neuwirth C, Tan Y, Durighel G, Cook SA. Assessment of severe reperfusion injury with $\mathrm{T} 2$ * cardiac MRI in patients with acute myocardial infarction. Heart 2010;96(23):1885-1891.

17. Husser O, Monmeneu JV, Sanchis J, et al. Cardiovascular magnetic resonance-derived intramyocardial hemorrhage after STEMI: influence on long-term prognosis, adverse left ventricular remodeling and relationship with microvascular obstruction. Int J Cardiol 2012. Jun 8. [Epub ahead of print]

18. Kali A, Kumar A, Cokic I, et al. Chronic manifestation of postreperfusion intramyocardial hemorrhage as regional iron deposition: a cardiovascular magnetic resonance study with ex vivo validation. Circ Cardiovasc Imaging 2013;6(2):218-228.

19. Mather AN, Fairbairn TA, Ball SG, Green wood JP, Plein S. Reperfusion haemorrhage as determined by cardiovascular MRI is a predictor of adverse left ventricular remodelling and markers of late arrhythmic risk. Heart 2011;97(6):453-459.

20. Eitel I, Kubusch K, Strohm O, et al. Prog nostic value and determinants of a hypointense infarct core in T2-weighted cardiac magnetic resonance in acute reperfused STelevation-myocardial infarction. Circ Cardiovasc Imaging 2011;4(4):354-362.

21. Ochiai K, Shimada T, Murakami Y, et al. Hemorrhagic myocardial infarction after coronary reperfusion detected in vivo by magnetic resonance imaging in humans: prevalence and clinical implications. J Cardiovasc Magn Reson 1999;1(3):247-256.

22. van den Bos EJ, Baks T, Moelker AD, et al. Magnetic resonance imaging of haemorrhage within reperfused myocardial infarcts: possible interference with iron oxide-labelled cell tracking? Eur Heart J 2006;27(13):1620-1626.

23. Kumar A, Green JD, Sykes JM, et al. Detection and quantification of myocardial reperfusion hemorrhage using $\mathrm{T} 2$ * weighted CMR. JACC Cardiovasc Imaging 2011;4(12):1274-1283.
24. Zia MI, Ghugre NR, Connelly KA, et al Characterizing myocardial edema and hemorrhage using quantitative T2 and $\mathrm{T} 2 *$ mapping at multiple time intervals post ST-segment elevation myocardial infarction. Circ Cardiovasc Imaging 2012;5(5):566-572.

25. Lotan CS, Bouchard A, Cranney GB, Bishop SP, Pohost GM. Assessment of postreperfusion myocardial hemorrhage using proton NMR imaging at 1.5 T. Circulation 1992;86(3):1018-1025.

26. Basso C, Corbetti F, Silva C, et al. Morphologic validation of reperfused hemor rhagic myocardial infarction by cardiovascular magnetic resonance. Am J Cardiol 2007;100(8):1322-1327.

27. Eitel I, Desch S, Fuernau G, et al. Prognostic significance and determinants of myocardial salvage assessed by cardiovascular magnetic resonance in acute reperfused myocardial infarction. J Am Coll Cardiol 2010;55(22):2470-2479.

28. Payne AR, Berry C, Kellman P, et al. Bright-blood T(2)-weighted MRI has high diagnostic accuracy for myocardial hemorrhage in myocardial infarction: a preclinica validation study in swine. Circ Cardiovasc Imaging 2011;4(6):738-745.

29. Bradley WG Jr. MR appearance of hemorrhage in the brain. Radiology 1993;189(1):15-26.

30. Lotan CS, Miller SK, Cranney GB, Pohost GM, Elgavish GA. The effect of postinfarction intramyocardial hemorrhage on transverse relaxation time. Magn Reson Med 1992;23(2):346-355.

31. Bondarenko O, Beek AM, Hofman MB, et al. Standardizing the definition of hyperenhancement in the quantitative assessment of infarct size and myocardial viability us ing delayed contrast-enhanced CMR. J Cardiovasc Magn Reson 2005;7(2):481-485.

32. O’Regan DP, Ahmed R, Karunanithy N, et al. Reperfusion hemorrhage following acute myocardial infarction: assessment with $\mathrm{T} 2 *$ mapping and effect on measuring the area at risk. Radiology 2009;250(3):916-922.

33. Jensen JH, Chandra R. NMR relaxation in tissues with weak magnetic inhomogeneities. Magn Reson Med 2000;44(1):144156.

34. Gillis P, Koenig SH. Transverse relaxation of solvent protons induced by magnetized spheres: application to ferritin, erythrocytes, and magnetite. Magn Reson Med $1987 ; 5(4): 323-345$.

35. Carpenter JP, He T, Kirk P, et al. On T2* magnetic resonance and cardiac iron. Circulation 2011;123(14):1519-1528 